\title{
Auditory and vestibular dysfunction associated with blast-related traumatic brain injury
}

\author{
Stephen A. Fausti, PhD; ${ }^{1}$ Debra J. Wilmington, PhD; ${ }^{1 *}$ Frederick J. Gallun, PhD; ${ }^{1}$ Paula J. Myers, PhD; ${ }^{2}$ \\ James A. Henry, PhD $^{1}$ \\ ${ }^{1}$ Department of Veterans Affairs (VA) Rehabilitation Research and Development Service, National Center for Rehabili- \\ tative Auditory Research, Portland VA Medical Center, Portland, OR; and Department of Otolaryngology, Oregon \\ Health \& Science University, Portland, OR; ${ }^{2}$ James A. Haley Veterans' Hospital/Polytrauma Rehabilitation Center, \\ Tampa, FL
}

\begin{abstract}
The dramatic escalation of blast exposure in military deployments has created an unprecedented amount of traumatic brain injury (TBI) and associated auditory impairment. Auditory dysfunction has become the most prevalent individual service-connected disability, with compensation totaling more than 1 billion dollars annually. Impairment due to blast can include peripheral hearing loss, central auditory processing deficits, vestibular impairment, and tinnitus. These deficits are particularly challenging in the TBI population, as symptoms can be mistaken for posttraumatic stress disorder, mentalhealth issues, and cognitive deficits. In addition, comorbid factors such as attention, cognition, neuronal loss, noise toxicity, etc., can confound assessment, causing misdiagnosis. Furthermore, some auditory impairments, such as sensorineural hearing loss, will continue to progress with age, unlike many other injuries. In the TBI population, significant clinical challenges are the accurate differentiation of auditory and vestibular impairments from multiple, many times overlapping, symptoms and the development of multidisciplinary rehabilitation strategies to improve treatment outcomes and quality of life for these patients.
\end{abstract}

Key words: auditory dysfunction, blast, central auditory processing, hearing loss, polytrauma, prevention, rehabilitation, tinnitus, traumatic brain injury, vestibular.

\section{INTRODUCTION}

Concurrent injuries to the auditory system as a result of acute blast trauma and resultant traumatic brain injury (TBI) accounted for one-quarter of all injuries among marines during Operation Iraqi Freedom through 2004the most common single injury type [1]. Blast-related TBI produces significantly greater rates of hearing loss

\footnotetext{
Abbreviations: $\mathrm{ABR}=$ auditory brainstem response, $\mathrm{ASSR}=$ auditory steady-state response testing, BPPV = benign paroxysmal positioning vertigo, $\mathrm{CAP}=$ central auditory processing, CNS = central nervous system, FM = frequency modulation, $\mathrm{LAV}=$ light-armored vehicle, $\mathrm{OAE}=$ otoacoustic emissions, PTM = Progressive Tinnitus Management, PTSD $=$ posttraumatic stress disorder, $\mathrm{SNHL}=$ sensorineural hearing loss, $\mathrm{TBI}=$ traumatic brain injury, $\mathrm{TM}=$ tympanic membrane, $\mathrm{VA}=$ Department of Veterans Affairs, VRT = vestibular rehabilitation therapy.

* Address all correspondence to Debra J. Wilmington, PhD; VA Rehabilitation Research and Development Service, National Center for Rehabilitative Auditory Research, 3710 SW US Veterans Hospital Road, Portland, OR 97239; 503-220-8262, ext 56446; fax: 503-220-3439.

Email: debra.wilmington@med.va.gov

DOI:10.1682/JRRD.2008.09.0118
} 
and tinnitus compared with non-blast-related TBI, affecting up to 60 percent of these patients [2]. In addition, a considerable population of blast-trauma patients with central auditory system injury may have been misdiagnosed because of the lack of assessment criteria for this population and because of logistical testing difficulties with polytrauma patients. Auditory dysfunction has become the most prevalent individual service-connected disability, with compensation totaling more than $\$ 1$ billion annually. Furthermore, some impairments, such as sensorineural hearing loss (SNHL), will continue to progress with age, unlike many other injuries. In the population with TBI, significant clinical challenges are the accurate differentiation of auditory and vestibular impairments from multiple, many times overlapping, symptoms and the development of multidisciplinary rehabilitation strategies to improve treatment outcomes and quality of life for these patients.

\section{HEARING LOSS ASSOCIATED WITH BLAST EXPOSURE}

Auditory system damage resulting from military activity can be caused by blast exposure, noise-induced damage from explosion or weapon firing (acoustic trauma), or ototoxic medications that are used during treatment of injuries and is frequently due to a combination of factors. Simultaneous exposure to noise and ototoxic agents, such as solvents during the blast exposure or medications, can cause synergistic interactions, increasing damage [3-4]. Ear and balance deficits are commonly overlooked in patients with polytrauma, and patients with TBI can be misdiagnosed as unresponsive when hearing loss is present [5]. Clinical reports of hearing loss from a patient usually do not occur until audibility within the frequency range important for speech understanding $(<8 \mathrm{kHz})$ is reduced and a communication problem becomes significant. In addition, damage to other neurological systems, such as those involved in memory and attention, can directly influence auditory system assessment and rehabilitation. Conversely, auditory system assessment may also be used to identify damage to other systems as a result of TBI (for example, a ruptured tympanic membrane [TM] has been used as a potential marker for concussive injury) [6-7]. Therefore, a comprehensive, multidisciplinary approach is essential for the evaluation and treatment of patients with TBI to minimize the consequences of blast injury.

\section{INJURIES TO AUDITORY SYSTEM}

Excessive noise exposure can damage the hair cells of the cochlea by creating vascular, metabolic, and chemical changes to normal cell processes, producing temporary or permanent threshold shifts. Noise can also cause a decrease in the stiffness of hair cell stereocilia and swelling of the auditory nerve endings, which affect the coupling and transfer of sound energy [8]. This damage leads to cell death and eventually to the degeneration of the auditory nerve fiber.

When exposed to the blast wave of an explosion, the human auditory system is vulnerable to both peripheral and central damage from the pressure wave itself and from the secondary and tertiary effects of blown objects impacting the body and the blown body impacting stationary objects. The increased duration and intensity of sounds endured in blast trauma typically affect both ears and will have a greater impact if directed laterally to the head. Permanent, pure SNHL is the most prevalent type of auditory impairment in blast trauma, accounting for 35 to 54 percent of injuries [2].

Rupture of the TM is the most frequent result of blast exposure, with clinical reports of incidence ranging from 4 to 79 percent [1,9-11]. TM rupture is also a clinical indicator that a patient has undergone a significant exposure and has been recommended as a diagnostic tool for determining whether or not life-threatening injuries may be present in blast survivors and as a potential marker for concussive injury [6]. The absence of TBI, however, should not be assumed when TMs are intact [7].

The most common otologic complaints immediately postblast are otalgia, tinnitus, aural fullness, dizziness, loudness sensitivity, distorted hearing, and hearing impairment. Concussions to the brain can cause audiologic symptoms such as hearing loss, dizziness, and central deficits because the auditory centers, such as the temporal lobe, corpus collosum, and thalamus, are vulnerable to damage. Temporal bone fractures can cause audiovestibular damage. The underlying symptoms of vestibular, peripheral, and central auditory damage can can overlap with those of posttraumatic stress disorder (PTSD), concussion, and mild TBI [12]. Postural instability and inner-ear dysfunction resulting from any of these 
conditions may persist up to 6 months or longer after blast trauma [13]. A multidisciplinary evaluation and long-term observation are therefore essential for the accurate diagnosis and treatment of patients with TBI.

\section{OUTER EAR INJURY}

The pinna can be burned and damaged by flying debris during blast exposures $[6,14]$. Hearing sensitivity can be preserved when the burn occurs without associated noise-induced hearing loss or penetrating trauma. Many burn patients, however, are treated with infectioncontrolling antibiotics (such as gentamicin, tobramycin, and amikacin) and therefore may be at risk for ototoxicity.

\section{MIDDLE EAR INJURY}

\section{Tympanic Membrane Perforation}

The TM is displaced and ruptures as a result of the primary shock wave traveling down the external auditory canal. While other organs in the body need pressure gradients of 56 to $76 \mathrm{lb} / \mathrm{in}^{2}$ for damage to occur [15], the ear is much more sensitive, with ruptures occurring in 50 percent of adults at $5 \mathrm{lb} / \mathrm{in}^{2}$ (approximately $185 \mathrm{~dB}$ peak pressure level) depending on the noise spectra and duration [16-17]. Explosives used in Operation Iraqi Freedom/Operation Enduring Freedom produce pressures that exceed $60 \mathrm{lb} / \mathrm{in}^{2}{ }^{2}$ and reach peak pressure in about 2.5 to $50 \mathrm{~ms}$. The rupturing of the TM can have a protective effect on the inner ear, however, as it dispels some of the sound energy that would be transmitted further into the ear [14]. Most TMs heal without surgical intervention, except in cases of resultant middle ear infection or when blast causes total TM perforation.

When small fragments of the squamous epithelium enter the middle ear cavity after a TM rupture, these cells can grow into cholesteatomas that can infect or erode ossicles. Cholesteatomas usually result in a conductive hearing loss and dizziness, and associated muscle weakness on that side can also occur. Recurrence of cholesteatomas can occur in 10 to 20 percent of cases, and complications result in nerve damage and deafness [16].

\section{Ossicular Disruption}

In cases of severe blast trauma, the pressure wave from blast trauma can disrupt the ossicles of the middle ear that transmit sound vibrations to the cochlea. Ossicular disruption results in a conductive hearing loss, and with surgical repair, patients regain much of their hearing at the lower frequencies [18]. Similar to the rupture of the $\mathrm{TM}$, ossicular damage may dispel some of the energy transmitted by the blast wave, preserving the inner ear. Conversely, pressure waves can cause inner-ear damage without affecting the ossicular chain.

\section{INNER EAR INJURY: COCHLEAR INJURY}

The blast wave and ensuing noise exposure cause structural damage to the inner and outer hair cells, resulting in conductive hearing loss and/or SNHL [19]. This damage is primarily mechanical, as the force of the blast wave tears the sensory cells and displaces the basilar membrane. Otologic injury is usually present at the time of the blast, producing temporary or permanent hearing loss and/or tinnitus [20]. Typically, sustained high-intensity noise causes more sensorineural damage than a single, very high-intensity blast [18]. The oval or round window can also be disrupted by the blast exposure, which can cause permanent hearing loss [21].

Blast exposure most commonly results in pure SNHL, with a 35 to 100 percent incidence rate in blastinjured patients [2,19,20,22-24]. Of blast-injured soldiers treated at Walter Reed Army Medical Center in 2005, 64 percent had ongoing hearing loss [5]. The audiometric configuration of hearing in those exposed to blast is a high-frequency hearing loss at one or more frequencies, although some can present with flat configurations, from mild to severe hearing loss or profound deafness. Although acoustic trauma typically results in a decrease in hearing sensitivity around $4 \mathrm{kHz}$ (a "noise notch"), blast injuries produce a sloping high-frequency hearing loss that often affects frequencies below $8 \mathrm{kHz}$. In addition, the initial presentation is frequently a mixed hearing loss (conductive and SNHL) due to middle ear injury, followed by at least partial recovery of conductive loss as injuries heal.

\section{OTOTOXICITY}

TBI patients often require treatment with drug regimens, such as aminoglycoside antibiotics for infection, that are ototoxic and cause irreversible cochlear and/or 
vestibular damage. Ototoxicity can occur in 60 to 70 percent of patients, resulting in hearing loss and tinnitus and often causing further impairment to an individual with a preexisting hearing loss [25]. Ototoxicity produces a bilateral SNHL that begins at the high frequencies and subsequently progresses to the lower frequencies [26-29]. Ototoxic damage to the auditory structures can also continue to progress after the drug treatment has ended. This progression of hearing loss is due to the continued preferential uptake of the ototoxic drug by the cells in the cochlea after the end of treatment and by slow clearance of the drug by the cells [30]. Previous noise exposure increases the risk of ototoxicity. Since patients with TBI typically have been exposed to excessive noise, these patients have an increased risk of ototoxic hearing loss and should be monitored [31]. Current recommendations for ototoxicity monitoring include the assessment of hearing thresholds at frequencies up to $12 \mathrm{kHz}$ or to the frequency limits of the individual's hearing [32]. These patients should be evaluated using conventional and high-frequency audiometry upon initial examination and be monitored serially for change in auditory sensitivity on a 5-day cycle throughout antibiotic therapy.

\section{AUDITORY EVALUATION}

Patients with TBI often have multiple injuries, varying levels of consciousness, and contraindications to testing that impede an auditory evaluation. Testing protocols frequently have to be modified to accommodate the patient's injuries. For example, headphones, insert earphones, or bone conduction may not be able to be used because of skull injury and/or burns. Objective measurements such as otoacoustic emissions (OAE), auditory steady-state response testing (ASSR), and auditory brainstem response (ABR) testing may be employed when behavioral response cannot be obtained or to confirm test results. In addition, modifications to existing protocols to compensate for cognitive impairments, such as the use of a slower rate of speech presentation in auditory tests, can facilitate auditory assessment. No standard test battery or procedures exist for the diagnosis of otologic, otovestibular, and central auditory processing (CAP) disorders in patients with TBI. Furthermore, because the long-term sequelae of TBI are not fully known, follow-up is essential in these patients.

Case history and other standardized questionnaires that assess the impact of hearing loss, tinnitus, and/or diz- ziness should be acquired from a TBI patient to elicit information about cognitive functioning and visual, audiologic, and tinnitus history both before and after the blast exposure. The Hearing Handicap Inventory for Adults assesses the emotional and social consequences of hearing loss [33]. Patients should also be asked to provide detailed information about their blast exposure, such as the nature of the blast, the proximity to the blast, the use of hearing protection, and the loss of consciousness at the time of injury. Patients with TBI are generally good historians regarding their preinjury capabilities, but supplemental sources of information are important as many patients lack current self-awareness and self-assessment skills or exhibit a hyperacute awareness of their symptoms.

A comprehensive audiometric evaluation should be completed, including otoscopy, air- and bone-conduction thresholds, immittance audiometry, ipsilateral and contralateral acoustic reflex thresholds, and contralateral acoustic reflex decay. Tests of speech reception thresholds and speech intelligibility for one-syllable words should be conducted. ABR and ASSR testing can be used if conventional behavioral audiometry is not possible. Results of OAE can verify other test results.

\section{TREATMENT FOR PERIPHERAL HEARING LOSS}

Hearing aids and other assistive-listening-device options can significantly improve a patient's ability to communicate by improving speech perception in everyday listening environments. Hearing aids that can automatically adjust to different acoustic environments may be especially suitable for patients with TBI with upperlimb amputation and/or cognitive disorders. Frequency modulation (FM) allows the speaker's voice to be picked up via a wireless microphone and then transmitted via FM radio waves from the transmitter to the receiver. Other options include television captioning, teletext or volume-control telephones, and alerting devices that employ flash-visual or vibrotactile signals to alert the person of acoustical environmental events.

\section{TINNITUS}

Tinnitus is defined as head or ear noise lasting 5 minutes or longer [34-35]. However, tinnitus is present most or all of the time for the typical patient with tinnitus [36]. 
Anything that causes damage to hearing acuity can also cause tinnitus [37]. In addition, tinnitus can be a symptom of other health problems, including stress; allergies; tumors; metabolic factors; and problems in the heart and blood vessels, jaws, and neck. The most common cause of tinnitus is excessive noise exposure [38-39]. Noiseinduced tinnitus is most commonly characterized as a high-pitched tonal or hissing sound [36]. Although tinnitus symptoms resulting from blast and/or impulse noise exposure may resolve, persistent symptoms occur in some patients, with many of these patients reporting greater disability from the tinnitus than from the associated hearing loss [40]. Cave et al. stated, "Tinnitus can be particularly prevalent for patients who suffer from it secondary to blast injury to their ears, because of the sudden onset of tinnitus in the case of blast injury, instead of the gradual onset of tinnitus developing slowly with progressive hearing loss” [41].

\section{TINNITUS MANAGEMENT}

Tinnitus patients have widely differing needs with regard to their tinnitus symptoms. Therefore, it is necessary to implement a hierarchical program of tinnitus management that is designed to be maximally efficient and have the least impact on clinical resources, while still addressing the needs of all patients who complain about tinnitus [42]. A hierarchical program has been developed-Progressive Tinnitus Management (PTM) - that involves five levels of clinical services: (1) triage, (2) audiologic assessment, (3) group educational counseling, (4) interdisciplinary evaluation, and (5) individualized support (repeated appointments to implement one-on-one care) [43-44]. The majority of tinnitus patients have their needs met by receiving the Level 2 audiologic assessment (including hearing aids, if necessary) and Level 3 group education, which ideally consists of group sessions conducted by an audiologist and additional sessions conducted by a psychologist. The group sessions provide patients with selfhelp strategies and general recommendations for minimizing tinnitus symptoms. Relatively few patients require the Level 4 interdisciplinary evaluation and Level 5 individualized support. Patients with a severe loudnesstolerance problem undergo a special treatment program that is associated with the PTM protocol. If the patient does not make adequate progress with PTM, then other forms of tinnitus therapy, such as tinnitus retraining therapy, neuromonics tinnitus treatment, or cognitive-behavioral therapy, can be used.

\section{CENTRAL AUDITORY SYSTEM DAMAGE}

The main focus of today's conventional auditory evaluation is on damage to the peripheral auditory system. One of the potential results of TBI, however, is damage to the central auditory system. Such damage would be evident as impairments in CAP, which can result in difficulty hearing in background noise, trouble with discrimination of temporal characteristics or auditory patterns necessary for speech perception, and difficulty with sound localization and lateralization [25]. This occult injury can easily go undiagnosed because of the focus of audiologic professionals on those aspects of hearing with which they are most familiar. One of the greatest challenges presented by TBI is the need for new ways of approaching familiar systems. The diagnosis, rehabilitation, and prevention of central auditory system damage is currently in its infancy, with only the barest indications of the directions in which it should be headed.

Patients exposed to blasts have a high chance of being hit by flying objects or of being picked up by the blast wave and colliding with a stationary object, such as a vehicle or a wall. In both cases, there is a significant chance that the brain suffered impact. It has been well established that when the brain moves within the skull to an extent that it impacts bone, contusions (hemorrhage and edema) occur, and damage to the central nervous system (CNS) after an explosion has been increasingly attributed to the direct effects of the blast [6]. An additional potential mechanism of blast injury to the central auditory system is the shearing, stretching, and/or angular forces that can be exerted on axons and small blood vessels, which can result in swelling and disconnection of axons [45]. While the proportion of those exposed to blasts that have damage to the central auditory system is not yet known, Taber et al. report that the auditory processing areas of the temporal lobe are among the most commonly affected regions [45].

The effects of damage to the central auditory system have not been systematically examined in humans, but animal studies have revealed much about the architecture of the system, from which it is possible to speculate about the sorts of deficits that would result [46-48]. The shearing or stretching forces from blast exposure can 
result in a disruption of the parallel organization of the brainstem through swelling, cell death, and/or disconnection of inputs to brainstem nuclei. One of the main roles of the auditory brainstem is to provide sensitivity to the fine temporal structure of auditory stimuli, allowing identification of pitch information and harmonic relationships and improving sound processing in the presence of interfering sounds. Any disruption of the delicate architecture of the connections throughout the brainstem can result in a loss of the precise temporal alignment of inputs necessary to perform these tasks. For example, damage to structures involved with comparing stimuli arriving from the two ears ("binaural" processing) can reduce the ability to localize sounds without producing any overt loss in sensitivity to tonal stimuli. Sheft and Yost review many of the important types of information conveyed by the temporal structure of sounds [49], and Darwin describes how binaural information can be used to distinguish among multiple sound sources and thus organize the auditory scene into meaningful units [50].

Brainstem damage can also disrupt connectivity to higher centers, potentially resulting in an impaired ability to construct meaningful units out of the complex information being passed from the cochlea. A common complaint from people with hearing impairment-that noisy or crowded rooms are the worst listening environmentscan be directly related to difficulties with binaural hearing and other types of temporal processing. To date, however, no large detailed studies have been performed with hearing-impaired listeners from which to draw firm conclusions, particularly when it comes to the importance of binaural hearing. No studies have been conducted on listeners with good detection thresholds and poor binaural hearing. Although the impact of these timing and binaural deficits in a real-world situation could be profound, tests of these abilities are rarely performed.

Very little is known about thalamic and cortical damage, but a number of reports indicate the crucial role such structures play in hearing. For example, "cortical deafness," in which immediate and ongoing insensitivity to all sounds is present, can result from bilateral damage to the primary auditory cortex (the superior temporal gyrus). In addition, a number of additional processing deficits (agnosias) are associated with damage to auditory cortical areas that involve comprehension of speech, appreciation and discrimination of music, and identification and discrimination of environmental sounds. The extent to which attentional and behavioral processes underlie these deficits is not yet well understood [51].

One of the main reasons that effective and widely adopted diagnostic tests and treatment regimens have not been developed is the multidisciplinary nature of the task to be accomplished. Both peripheral and central auditory deficits need to be identified to determine precisely where the damage has occurred. Currently, only a few tests are available to assess central damage, and even for those that exist, the relationship between test results and specific dysfunction is tenuous. The collaboration of auditory researchers with cognitive psychologists and neurologists will significantly benefit the patient with TBI.

Even if it is assumed that basic research will eventually determine the links between specific types of damage to the auditory system and the outcome of particular tests, there is still the hurdle of developing clinically usable tests that are specific and reliable to CAP deficits. Such an endeavor requires the translation of basic research into clinical practice and is prone to one of the most fundamental issues in translational research: pinpointing the time when the basic science has progressed sufficiently to develop a clinical version of a given test. Finally, research efforts must also develop and confirm the effectiveness of a rehabilitative therapy. Until the multidisciplinary investigative teams can be assembled to accomplish these tasks, it will not be possible to deliver the most efficacious tools and methods required to accurately diagnose and treat CAP deficits in adults who have suffered TBI.

Despite these hurdles, however, it is important to point out that the auditory system has great promise as a "canary in the mine" for those patients whose brain injury is not great enough to be easily identified. In the veteran population, such patients may be misdiagnosed with PTSD, which can lead to inappropriate therapies and increase frustration and emotional distress for the injured patient. Through the development of rapid protocols and portable testing equipment, the auditory modality can be used to test for fundamental processing dysfunction immediately after exposure to a blast or other potentially brain-damaging event.

\section{Diagnosis of Central Auditory System Damage}

Central auditory deficits are one of many types of damage caused by injury to the brain and can be mistaken for PTSD, mental-health issues, and cognitive deficits. The already complex task of CAP assessment is even 
more difficult in the TBI population because variables such as motivation, attention, cooperation, cognition, neuronal loss, noise toxicity, metabolic and circulatory changes, working memory, and other comorbid factors can confound test interpretations and cause misdiagnosis. Anecdotal evidence from Department of Veterans Affairs (VA) audiologists suggests that a significant number of those who have been exposed to blasts during combat have normal hearing sensitivity, yet still report difficulties hearing in noise. Although these symptoms may be attributed to frontal lobe damage resulting in attention deficits, this type of hearing impairment is expected when damage occurs to central processing areas crucial to sound source segregation and the ability to focus on a single target among distractors. As Lux has pointed out, "persons who have sustained a significant TBI can look strikingly normal in a quiet, structured clinic or even a neuropsychology laboratory, yet break down cognitively or behaviorally once they have to interact with the uncertainties and distractions of the real word" [52]. This dichotomy is even more likely to occur for patients who have suffered a moderate or severe TBI because they may be unaware that they have a hearing impairment. Conversely, persons with mild TBI frequently report hyperacute sensory awareness. At the current time, insufficient research is available to indicate which of the multiple tests of central processing are most appropriate for identifying these patients. Nor is there a way to clearly identify a course of rehabilitation that has been shown to improve performance.

Some potential test measures that an audiologist can consider for inclusion in a CAP assessment battery for a given patient are auditory temporal processing and patterning tests; dichotic speech tests; monaural lowredundancy speech tests; binaural interaction tests; and electrophysiologic tests, such as the middle-latency response and the late-latency response, to reflect activity generated by the CNS in response to auditory stimulation. Neuropsychologists also use measures of attention, processing speed, and memory to assess the presence of CAP deficits.

\section{Treatment of CAP Dysfunction}

Treatment options for CAP deficits are currently under investigation by the research community. Current clinical guidelines recommend a two-step approach that includes auditory training as well as general management options [25]. Auditory training is designed to capitalize on the plasticity of the auditory system by altering the neural encoding of sound and subsequent timing of brainstem responses. Subjects can learn to interpret as speech sounds that could not be discriminated before training. It is recommended that training occur soon after injury to maximize the plasticity of the brain and that training be patient-specific and focused on the deficit areas noted on the CAP assessment test battery. General management strategies include use of environmental strategies, such as an FM system, and teaching of compensatory strategies. Deficit-specific remediation activities such as phonological awareness and discrimination training (speech to print skills), auditory closure, prosody training, speech reading, auditory training exercises, and exercises to improve interhemispheric transfer of information via cross-modality activities can be used.

\section{VESTIBULAR INJURY}

\section{Labyrinthine Damage}

The vestibular system consists of the semicircular canals and otolith organs in the inner ears, which provide information to the brain about head movement and head position to maintain balance and postural stability. Nerve connections from the vestibular sensory organs provide information about head movement that results in eye movements that keep vision stable when the head moves and in posture changes in the neck, trunk, arms, and legs that maintain balance. While nonvestibular causes such as visual impairment, central pathology, medications, and proprioceptive changes can cause dizziness and imbalance, head injury can cause a loss of peripheral vestibular function. Dizziness and balance problems can occur with blast exposure and TBI, and their presence is a prognostic indicator in this population. Balance problems are less frequent and well defined than auditory symptoms, however, occurring in 15 to 78 percent of patients with mild head injuries [53-54].

The effects of blast exposure on the vestibular system are not well understood. Some investigators have suggested that dizziness and imbalance are a result of CNS damage caused by TBI [55-56]; however, dizziness and imbalance can result from damage by the blast wave to the peripheral vestibular system and not from the head injury itself. Vestibular injury can occur to one or both ears and may affect the sensory organs, the vestibular nerve, or other components of the vestibular pathway. In 
one of the few long-term studies on untreated patients with mild head trauma injury, vertigo persisted in 59 percent of patients after 5 years of recovery [57]. Patients with mild TBI who have symptoms of dizziness and imbalance often experience a slower recovery and are less likely to return to work than patients without dizziness [58].

Head trauma as a result of blast injury is one of the most frequent causes of benign paroxysmal positioning vertigo (BPPV), the most common balance disorder of head trauma patients, with an occurrence rate of 10 to 25 percent $[13,35,59-60]$. BPPV is characterized by brief (a few seconds or a minute) severe vertigo associated with changing head positions, such as looking up or rolling over in bed. Labyrinthine damage may also occur after blast injuries, resulting in complaints of vertigo.

Some acoustic trauma and vertigo/dizziness studies also suggest that there may be balance disturbances resulting from noise exposure [61]. Asymmetric exposure to extremely intense ( $\geq 140 \mathrm{~dB}$ ) sounds leads to a greater likelihood of vertigo. Furthermore, patients with TBI may also have been exposed to toxic chemicals, which can affect the CNS and which may present as vertigo or unsteadiness [62]. Finally, ototoxicity due to medications used in the treatment of conditions associated with head trauma can on rare occasions cause temporary or permanent balance loss, hearing loss, or both.

Many patients do well with a compensated unilateral peripheral lesion. Patients with uncompensated unilateral peripheral or bilateral damage to the vestibular system, however, must depend on other components of the sensory system (vision and proprioception) [63]. For the polytrauma patient with visual field deficit and lowerlimb loss, this complicates the rehabilitation process. Whereas central compensation will typically result in a decrease of balance issues over time, patients with TBI present a particular challenge, since symptoms sometimes continue for 6 months or longer.

\section{Central Vestibular Damage}

Effects of otologic blast injuries can include central vestibular pathology, although these disorders are less common than peripheral damage in patients following head injury [35] and blast exposure [24]. In some cases, there may be evidence of both central and peripheral vestibular dysfunction [35]. Central vestibular trauma may be due to postconcussive syndrome or result from cerebral or brainstem injuries $[61,64]$.

\section{Vestibular Evaluation}

If the patient reports any problems with dizziness or unsteadiness, balance testing should be conducted. Balance is a multisensory function; thus, evaluations are not sensitive to site-of-lesion tests. Clinicians working with individuals with blast trauma need to consider several causes of postural instability, including TBI, orthostatic hypotension, cervical vertigo, visual deficits, possible side effects of ototoxic drugs, and vestibular pathology. Screening tests typically administered by physical therapists during the vestibular portion of the blast evaluation include a cervical assessment, oculomotor evaluation, postural stability, gait assessments, and a vertebral artery test to assess for potential vertebral insufficiency and vascular causes of dizziness. Screening tests are not sensitive enough to detect all balance problems, however, and do not provide quantitative data to determine the presence or absence of vestibular lesions. The Dizziness Handicap Inventory assesses handicap by quantifying the functional, emotional, and physical effects of dizziness and unsteadiness.

Tests of peripheral vestibular function include horizontal semicircular canal function tests, such as caloric and rotational testing, and otolith testing, such as vestibular-evoked myogenic potentials. More recent best practices have also incorporated the computerized dynamic visual acuity test $[63,65]$ and the subjective visual vertical test during off-axis rotation as a test of utricle function [66] in the blast-injured population. BPPV should be ruled out as a cause of dizziness by performing the DixHallpike and roll tests. The presence or absence of spontaneous and/or positional nystagmus should be observed as an indicator of vestibular adaptation. Tests to assess central vestibular function include ocular motor tests and tests of vestibular suppression.

\section{Treatment of Vestibular System}

Treatment of balance problems should be patient and deficit specific and should be provided in collaboration with both physicians and physical therapists. Depending on diagnostic test results, treatment options can include canalith repositioning therapy for treatment of BPPV, vestibular rehabilitation therapy (VRT) for patients with balance and/or horizontal semicircular canal dysfunction, physical therapy, change in activity levels, medication, change in diet, treatment for any underlying disease that may be contributing to the balance disorder (such as eye glasses, infection, etc.), or surgery. Otolith disorders are more difficult to treat as little is known regarding the 
adaptation process that occurs following otolith damage. Basta et al. have shown that traditional VRT is not effective for many patients with otolith disorders [67].

\section{PREVENTION}

Because of the many types of ear injuries incurred by soldiers, multiple strategies to prevent auditory impairment must be employed. Physical barriers to sound, pressure, and debris in the form of headphones and earplugs prevent injury to the external and middle ear. Technological advances have enabled hearing protectants to be equipped with microphones that maintain hearing sensitivity while offering maximum safety. For those wearing hearing protection, the risk of hearing loss is minimized and the incidence of ruptured TM is significantly reduced. In many incidents in which troops used hearing protection when exposed to blasts, they did not sustain ear injuries, whereas soldiers without ear protection experienced ear damage and hearing loss [68]. Military personnel assigned to light-armored vehicles (LAVs) as commanders, gunners, and drivers wear protective helmets. Personnel in the rear of the vehicle do not generally wear protection, however, and therefore are most susceptible to incurring ear blast injuries [1]. An evaluation of blastrelated injuries in soldiers traveling in LAVs from multiple military conflicts reported that more than 20 percent of wounded soldiers presented with ear injury, mostly ruptured TMs [1]. In contrast, eye injuries are extremely uncommon for these soldiers, presumably because of the nearly 100 percent conformity to ballistic eye protection use by soldiers traveling in LAVs [1]. The adoption of hearing-protection standards is therefore essential to shield soldiers potentially at risk for blast or excessive noise exposure.

Hearing protection will not, however, provide protection against central auditory damage resulting from TBI. Improvements to protective head gear will need to be combined with ear protection to minimize auditory system damage. In addition, monitoring of hearing thresholds while soldiers are in the field, as well as when patients are receiving ototoxic medications, will provide for early identification of hearing loss. The development of field assessment tools for central auditory and vestibular damage will also identify problems early and maximize treatment options.
Systemic compounds for otoprotection may also prevent damage at the cellular level. Otoprotective agents may be capable of preventing noise-induced and ototoxic hearing loss by reducing the damaging effects of noise or medications on the hair cells of the cochlea. In addition, some otoprotectants may be able to rescue damaged cells once injury has occurred, thereby minimizing hearing loss. There have been numerous studies of various compounds used to protect hearing loss, ranging from antioxidants that scavenge free radicals to agents that increase blood flow and drugs that block cell-death-pathway signaling factors [69-71]. Despite the potential these investigations bring for protection from hearing damage, the translation of animal studies of otoprotectants to clinical treatment has been limited. Clinical trials are needed to delineate the effects of these protective compounds. Effective preventative therapies may ultimately require a combination of compounds that employ multiple mechanisms to combat cell damage in the inner ear. Early identification and immediate treatment of auditory system damage will ultimately lead to improved rehabilitative outcomes, reduced cognitive deficits, and improved quality of life [72].

\section{CONCLUSIONS}

The dramatic escalation of blast exposure has created an unprecedented amount of documented brain injury and associated auditory impairment and raises questions about additional patients with TBI that may be misdiagnosed or missed. It is critical that appropriate central auditory assessment tools and techniques be developed and utilized by a multidisciplinary team to comprehensively assess peripheral, central, and vestibular components of the auditory system.

Currently, auditory rehabilitation has been an endorgan discipline. The treatment and rehabilitation of auditory and vestibular injury associated with blast trauma, however, will require a multidisciplinary approach including otolaryngology, neurology, audiology, speech-language pathology, neuropsychology, mental health, and physical and occupational therapy. The type of auditory deficit present in the patient will determine treatment options that include medical/surgical interventions, technological considerations, and auditory training/counseling. In addition, patients with TBI may have cognitive impairments (problems with orientation, attention, concentration, 
perception, comprehension, learning, organization of thought, executive function, problem solving, and memory) that will require special attention to maximize the benefits of rehabilitation. Providers may need to introduce new ideas and concepts by showing the patient examples. Reducing visual and auditory distractions and giving directions one step at a time are also ways that providers can reduce confusion for a patient with cognitive impairments. It is also important to demonstrate and explain information in more than one modality. Although eye injuries are less common in LAV accidents, up to 28 percent of soldiers exposed to blast have significant eye injuries. Visual-field deficits must be taken into consideration when providing aural rehabilitation and amplification device instructions. Patients may have limitations in their field of vision and require the use of glasses, magnifiers, or other visual aids to benefit from rehabilitation. Furthermore, behavioral and emotional issues, including aggression, agitation, mental trauma, and adjustment to disabilities, may interfere with treatment plans at early stages of recovery [73].

A comprehensive evaluation must be completed in polytrauma patients to ensure that all injuries are accurately diagnosed and appropriate rehabilitation can be devised. Furthermore, the implementation of hearing conservation strategies including physical ear protection, preventative therapy with otoprotectants, and the implementation of hearing loss early detection and monitoring programs may significantly reduce the number of patients who experience disabling hearing impairments. Effective treatment and hearing loss protection programs can also reduce the potential for the medical, legal, and socioeconomic consequences of hearing loss and ultimately allow patients to retain a better posttreatment quality of life.

\section{ACKNOWLEDGMENTS}

\section{Author Contributions:}

Study concept and design: S. A. Fausti, D. J. Wilmington,

F. J. Gallun, P. J. Myers, J. A. Henry.

Acquisition of data: S. A. Fausti, D. J. Wilmington,

F. J. Gallun, P. J. Myers, J. A. Henry.

Analysis and interpretation of data: S. A. Fausti,

D. J. Wilmington, F. J. Gallun, P. J. Myers, J. A. Henry.

Drafting of manuscript: D. J. Wilmington.

Critical revision of manuscript for important intellectual content:

S. A. Fausti, D. J. Wilmington, F. J. Gallun, P. J. Myers, J. A. Henry.
Administrative, technical, or material support: S. A. Fausti, D. J. Wilmington, F. J. Gallun, P. J. Myers, J. A. Henry.

Study supervision: S. A. Fausti.

Financial Disclosures: The authors have declared that no competing interests exist.

Funding/Support: This material was based on work supported by the VA Rehabilitation Research and Development Service (grants C4844C, B5067R, C4488R, C6770R, C4488R, CDA2 C4963W) and the National Center for Rehabilitative Auditory Research.

\section{REFERENCES}

1. Gondusky JS, Reiter MP. Protecting military convoys in Iraq: An examination of battle injuries sustained by a mechanized battalion during Operation Iraqi Freedom II. Mil Med. 2005;170(6):546-49. [PMID: 16001610]

2. Lew HL, Jerger JF, Guillory SB, Henry JA. Auditory dysfunction in traumatic brain injury. J Rehabil Res Dev. 2007; 44(7):921-28. [PMID: 18075949]

DOI:10.1682/JRRD.2007.09.0140

3. Fechter LD, Gearhart C, Fulton S, Campbell J, Fisher J, Na K, Cocker D, Nelson-Miller A, Moon P, Pouyatos B. JP-8 jet fuel can promote auditory impairment resulting from subsequent noise exposure in rats. Toxicol Sci. 2007;98(2): 510-25. [PMID: 17483120] DOI:10.1093/toxsci/kfm101

4. Brown JJ, Brummett RE, Meikle MB, Vernon J. Combined effects of noise and neomycin. Cochlear changes in the guinea pig. Acta Otolaryngol. 1978;86(5-6):394-400. [PMID: 716862]

5. Chandler D. Blast-related ear injury in current U.S. military operations. ASHA Leader. 2006;11(9):8-9,29.

6. DePalma RG, Burris DG, Champion HR, Hodgson MJ. Blast injuries. N Engl J Med. 2005;352(13):1335-42.

[PMID: 15800229]

DOI:10.1056/NEJMra042083

7. Xydakis MS, Bebarta VS, Harrison CD, Conner JC, Grant GA, Robbins AS. Tympanic-membrane perforation as a marker of concussive brain injury in Iraq. $\mathrm{N}$ Engl $\mathrm{J}$ Med. 2007;357(8):830-31. [PMID: 17715421] DOI:10.1056/NEJMc076071

8. Noise and hearing loss. NIH Consens Statement. 1990; 8(1):1-24.

9. Roth Y, Kronenberg J, Lotem S, Leventon G. [Blast injury of the ear]. Harefuah. 1989;117(10):297-310. Hebrew. [PMID: 2613087]

10. Lucic M. Eardrum perforation in explosion survivors: Is it a marker of pulmonary blast injury? Ann Emerg Med. 1995; 4:168-72.

11. De Ceballos JP, Turégano-Fuentes F, Perez-Diaz D, SanzSanchez M, Martin-Llorente C, Guerrero-Sanz JE. 11 March 2004: The terrorist bomb explosions in Madrid, 
Spain-An analysis of the logistics, injuries sustained and clinical management of causalities treated at the closest hospital. Crit Care. 2005;9(1):104-11. [PMID: 15693992] DOI:10.1186/cc2995

12. Kennedy JE, Jaffee MS, Leskin GA, Stokes JW, Leal FO, Fitzpatrick PJ. Posttraumatic stress disorder and posttraumatic stress disorder-like symptoms and mild traumatic brain injury. J Rehabil Res Dev. 2007:44(7):895-920.

[PMID: 18075948$]$

DOI:10.1682/JRRD.2006.12.0166

13. Cohen JT, Ziv G, Bloom J, Zikk D, Rapoport Y, Himmelfarb MZ. Blast injury of the ear in a confined space explosion: Auditory and vestibular evaluation. Isr Med Assoc J. 2002;4(7):559-62. [PMID: 12120473]

14. Helling ER. Otologic blast injuries due to the Kenya embassy bombing. Mil Med. 2004;169(11):872-76. [PMID: 15605933$]$

15. Katz E, Ofek B, Adler J, Abramowitz HB, Krausz MM. Primary blast injury after a bomb explosion in a civilian bus. Ann Surg. 1989;209(4):484-88. [PMID: 2930293] DOI:10.1097/00000658-198904000-00016

16. Kronenberg J, Ben-Shoshan J, Modan M, Leventon G. Blast injury and cholesteatoma. Am J Otol. 1988;9(2): 127-30. [PMID: 3407744$]$

17. Zajtchuk JT, Philips YY. Effects of blast overpressure on the ear. Ann Otol Rhinol Laryngol Suppl. 1989;140:5. [PMID: 2497692]

18. Chandler DW, Edmond CV. Effects of blast overpressure on the ear: Case reports. J Am Acad Audiol. 1997;8(2):81-88. [PMID: 9101454$]$

19. American Academy of Pediatrics. Pediatric terrorism and disaster preparedness: A resource for pediatricians. Foltin GL, Schonfeld DJ, Shannon MW, editors. AHRQ Publication No. 06(07)-0056. Rockville (MD): Agency for Healthcare Research and Quality. October 2006. Available from: http://www.ahrq.gov/research/pedprep/.

20. Mrena R, Pääkkönen R, Bäck L, Pirvola U, Ylikoski J. Otologic consequences of blast exposure: A Finnish case study of a shopping mall bomb explosion. Acta Otolaryngol. 2004;124(8):946-52. [PMID: 15513532] DOI:10.1080/00016480310017045

21. American College of Emergency Physicians. Emergency medicine: A comprehensive study guide. Tintinalli JE, Kelen GD, Stapczynski JS, editors. 5th ed. New York (NY): McGraw-Hill; 2000. p. 1275-77.

22. Persaud R, Hajioff D, Wareing M, Chevretton E. Otological trauma resulting from the Soho Nail Bomb in London, April 1999. Clin Otolaryngol Allied Sci. 2003;28(3):203-6. [PMID: 12755756$]$

DOI:10.1046/j.1365-2273.2003.00688.x
23. Perez R, Gatt N, Cohen D. Audiometric configurations following exposure to explosions. Arch Otolaryngol Head Neck Surg. 2000;126(10):1249-52. [PMID: 11031413]

24. Van Campen LE, Dennis JM, Hanlin RC, King SB, Velderman AM. One-year audiologic monitoring of individuals exposed to the 1995 Oklahoma City bombing. J Am Acad Audiol. 1999;10(5):231-47. [PMID: 10331616]

25. American Speech-Language-Hearing Association. Guidelines for the audiologic management of individuals receiving cochleotoxic drug therapy. Am Speech Lang Hear Assoc. 1994. Available from: http://www.asha.org/policy/.

26. Fausti SA, Henry JA, Schaffer HI, Olson DJ, Frey RH, McDonald WJ. High-frequency audiometric monitoring for early detection of aminoglycoside ototoxicity. J Infect Dis. 1992;165(6):1026-32. [PMID: 1583319]

27. Fausti SA, Henry JA, Schaffer HI, Olson DJ, Frey RH, Bagby GC Jr. High-frequency monitoring for early detection of cisplatin ototoxicity. Arch Otolaryngol Head Neck Surg. 1993;119(6):661-66. [PMID: 8499098]

28. Fausti SA, Larson VD, Noffsinger D, Wilson RH, Phillips DS, Fowler CG. High-frequency audiometric monitoring strategies for early detection of ototoxicity. Ear Hear. 1994;15(3):232-39. [PMID: 8076721] DOI:10.1097/00003446-199406000-00004

29. Macdonald MR, Harrison RV, Wake M, Bliss B, Macdonald RE. Ototoxicity of carboplatin: Comparing animal and clinical models at the Hospital for Sick Children. J Otolaryngol. 1994;23(3):151-59. [PMID: 8064951]

30. Aran JM, Chappert C, Dulon D, Erre JP, Aurousseau C. Uptake of amikacin by hair cells of the guinea pig cochlea and vestibule and ototoxicity: Comparison with gentamicin. Hear Res. 1995;82(2):179-83. [PMID: 7775283] DOI:10.1016/0378-5955(94)00175-P

31. Bokemeyer C, Berger CC, Hartmann JT, Kollmannsberger C, Schmoll HJ, Kuczyk MA, Kanz L. Analysis of risk factors for cisplatin-induced ototoxicity in patients with testicular cancer. Br J Cancer. 1998;77(8):1355-62. [PMID: 9579846]

32. Fausti SA, Erickson DA, Frey RH, Rappaport BZ, Schechter MA. The effects of noise upon human hearing sensitivity from 8000 to 20000 Hz. J Acoust Soc Am. 1981; 69(5):1343-47. [PMID: 7240565]

DOI:10.1121/1.385805

33. Newman CW, Weinstein BE, Jacobson GP, Hug GA. The Hearing Handicap Inventory for Adults: Psychometric adequacy and audiometric correlates. Ear Hear. 1990;11(6): 430-33. [PMID: 2073976]

DOI:10.1097/00003446-199012000-00004

34. Coles RR. Epidemiology of tinnitus: 2. Demographic and clinical features. J Laryngol Otol Suppl. 1984;9:195-202.

35. Davies RA, Luxon LM. Dizziness following head injury: A neuro-otological study. J Neurol. 1995;242(4):222-30. 
[PMID: 7798121]

DOI:10.1007/BF00919595

36. Meikle MB, Stewart BJ, Griest SE, Martin WH, Henry JA, Abrams HB, McArdle R, Newman CW, Sandridge SA. Assessment of tinnitus: Measurement of treatment outcomes. Prog Brain Res. 2007;166:511-21.

[PMID: 17956815]

DOI:10.1016/S0079-6123(07)66049-X

37. Dobie RA. Overview: Suffering from tinnitus. In: Snow JB, editor. Tinnitus: Theory and management. Lewiston (NY): BC Decker; 2004. p. 1-7.

38. Axelsson A, Barrenas ML. Tinnitus in noise-induced hearing loss. In: Dancer AL, Henderson D, Salvi RJ, Hamnernik RP, editors. Noise-induced hearing loss. St. Louis (MO): Mosby-Year Book; 1992. p. 269-76.

39. Penner MJ, Bilger RC. Psychophysical observations and the origin of tinnitus. In: Vernon JA, Moller RA, editors. Mechanisms of tinnitus. Needham Heights (MA): Allyn and Bacon; 1995. p. 219-30.

40. Mrena R, Savolainen S, Kuokkanen JT, Ylikoski J. Characteristics of tinnitus induced by acute acoustic trauma: A long-term follow-up. Audiol Neurootol. 2002;7(2):122-30. [PMID: 12006740]

DOI:10.1159/000057660

41. Cave KM, Cornish EM, Chandler DW. Blast injury of the ear: Clinical update from the global war on terror. Mil Med. 2007;172(7):726-30. [PMID: 17691685]

42. Henry JA, Schechter MA, Loovis CL, Zaugg TL, Kaelin C, Montero M. Clinical management of tinnitus using a "progressive intervention" approach. J Rehabil Res Dev. 2005; 42(4 Suppl 2):95-116. [PMID: 16470467]

DOI:10.1682/JRRD.2005.01.0005

43. Henry JA, Zaugg TL, Myers PJ, Schechter MA. Using therapeutic sound with progressive audiologic tinnitus management. Trends Amplif. 2008;12(3):188-209.

[PMID: 18664499]

DOI:10.1177/1084713808321184

44. Henry JA, Zaugg TL, Myers PJ, Schechter MA. The role of audiologic evaluation in progressive audiologic tinnitus management. Trends Amplif. 2008;12(3):170-87.

[PMID: 18628281]

DOI:10.1177/1084713808319941

45. Taber KH, Warden DL, Hurley RA. Blast-related traumatic brain injury: What is known? J Neuropsychiatry Clin Neurosci. 2006;18(2):141-45. [PMID: 16720789]

DOI:10.1176/appi.neuropsych.18.2.141

46. Joris PX, Schreiner CE, Rees A. Neural processing of amplitude-modulated sounds. Physiol Rev. 2004;84(2): 541-77. [PMID: 15044682] DOI:10.1152/physrev.00029.2003

47. Kaas JH, Hackett TA. Subdivisions of auditory cortex and processing streams in primates. Proc Natl Acad Sci U S A.
2000;97(22):11793-99. [PMID: 11050211]

DOI:10.1073/pnas.97.22.11793

48. Mickey BJ, Middlebrooks JC. Representation of auditory space by cortical neurons in awake cats. J Neurosci. 2003; 23(25):8649-63. [PMID: 14507964]

49. Sheft S, Yost WA. Discrimination of starting phase with sinusoidal envelope modulation. J Acoust Soc Am. 2007; 121(2):EL84-89. [PMID: 17348551] DOI:10.1121/1.2430766

50. Darwin CJ. Listening to speech in the presence of other sounds. Philos Trans R Soc Lond B Biol Sci. 2008; 363(1493):1011-21. [PMID: 17827106]

DOI:10.1098/rstb.2007.2156

51. Petkov CI, Kang X, Alho K, Bertrand O, Yund EW, Woods DL. Attentional modulation of human auditory cortex. Nat Neurosci. 2004;7(6):658-63. [PMID: 15156150] DOI:10.1038/nn1256

52. Lux WE. A neuropsychiatric perspective on traumatic brain injury. J Rehabil Res Dev. 2007;44(7):951-62.

[PMID: 18075952] DOI:10.1682/JRRD.2007.01.0009

53. Fitzgerald, DC. Head trauma: Hearing loss and dizziness. J Trauma. 1996;40(3):488-96. [PMID: 8601878$]$ DOI:10.1097/00005373-199603000-00034

54. Bergemalm PO, Borg E. Long-term objective and subjective audiologic consequences of closed head injury. Acta Otolaryngol. 2001;121(6):724-34. [PMID: 11678172] DOI:10.1080/00016480152583674

55. Kerr AG, Byrne JE. Concussive effects of bomb blast on the ear. J Laryngol Otol. 1975;89(2):131-43.

[PMID: 1123564] DOI:10.1017/S002221510008018X

56. Pahor AL. The ENT problems following the Birmingham bombings. J Laryngol Otol. 1981;95(4):399-406.

[PMID: 7229522] DOI:10.1017/S0022215100090873

57. Berman JM, Fredrickson JM. Vertigo after head injuryA five year follow-up. J Otolaryngol. 1978;7(3):237-45. [PMID: 151151]

58. Chamelian L, Feinstein A. Outcome after mild to moderate traumatic brain injury: The role of dizziness. Arch Phys Med Rehabil. 2004;85(10):1662-66. [PMID: 15468028] DOI:10.1016/j.apmr.2004.02.012

59. Proctor B, Gurdjian ES, Webster JE. The ear in head trauma. Laryngoscope. 1956;66(1):16-59. [PMID: 13287138] DOI:10.1288/00005537-195601000-00002

60. Barber HO. Positional nystagmus, especially after head injury. Laryngoscope. 1964;73:891-944. [PMID: 14178860]

61. Furman JM, Whitney SL. Central causes of dizziness. Phys Ther. 2000;80(2):179-87. [PMID: 10654064$]$ 
62. Alberti PW. Noise induced hearing loss. BMJ. 1992; 304(6826):522. [PMID: 1559054] DOI:10.1136/bmj.304.6826.522

63. Herdman SJ. Advances in the treatment of vestibular disorders. Phys Ther. 1997;77(6):602-18. [PMID: 9184686]

64. Hoffer ME, Gottshall KR, Moore R, Balough BJ, Wester D. Characterizing and treating dizziness after mild head trauma. Otol Neurotol. 2004;25(2):135-38. [PMID: 15021772] DOI:10.1097/00129492-200403000-00009

65. Herdman SJ, Schubert MC, Tusa RJ. Contribution of central pre-programming to visual acuity during head movements in patients with vestibular hypofunction. Arch Otolaryngol Head Neck Surg. 2001;127:1205-10.

66. Basta D, Todt, I, Scherer H, Clarke A, Ernst, A. Postural control in otolith disorders. Hum Mov Sci. 2005;24(2): 268-79. [PMID: 15953652] DOI:10.1016/j.humov.2005.04.002

67. Basta D, Singbartl F, Todt I, Clarke A, Ernst A. Vestibular rehabilitation by auditory feedback in otolith disorders. Gait Posture. 2008;28(3):397-404. [PMID: 18343666] DOI:10.1016/j.gaitpost.2008.01.006

68. Schulz TY. Troops return with alarming rates of hearing loss. Hearing Health. 2004;20:18-21.

69. Kopke R, Bielefeld E, Liu J, Zheng J, Jackson R, Henderson D, Coleman JK. Prevention of impulse noise-induced hearing loss with antioxidants. Acta Otolaryngol. 2005; 125(3):235-43. [PMID: 15966690] DOI:10.1080/00016480410023038

70. Kovacic P, Somanathan R. Ototoxicity and noise trauma: Electron transfer, reactive oxygen species, cell signaling, electrical effects, and protection by antioxidants: Practical medical aspects. Med Hypotheses. 2008;70(5):914-23.

[PMID: 17977665]

DOI:10.1016/j.mehy.2007.06.045

71. Campbell KC, Meech RP, Klemens JJ, Gerberi MT, Dyrstad SS, Larsen DL, Mitchell DL, El-Azizi M, Verhulst SJ, Hughes LF. Prevention of noise- and drug-induced hearing loss with D-methionine. Hear Res. 2007;226(1-2): 92-103. [PMID: 17224251]

DOI:10.1016/j.heares.2006.11.012

72. Arlinger S. Negative consequences of uncorrected hearing loss-A review. Int J Audiol. 2003;42 Suppl 2:2S17-20. [PMID: 12918624$]$

73. Cornis-Pop M. Blast injuries: A new kind of patient for speech-language pathologists. ASHA Leader. 2006;11(9): 6-7,28.

Submitted for publication September 2, 2008. Accepted in revised form January 21, 2009. 